\title{
Intravenous Immunoglobulin as Salvage Therapy in Refractory Pyoderma Gangrenosum: Report of a Case and Review of the Literature
}

\author{
John Cafardi ${ }^{a}$ Naveed Sami ${ }^{b}$ \\ aniversity of Cincinnati and The Christ Hospital, Cincinnati, Ohio, and ${ }^{b}$ University of \\ Alabama at Birmingham, Birmingham, Ala., USA
}

\section{Key Words}

Intravenous immunoglobulin $\cdot$ Treatment $\cdot$ Pyoderma gangrenosum

\begin{abstract}
Pyoderma gangrenosum is a neutrophilic dermatosis that occurs both as a primary disorder as well as secondary to an underlying disease. Due to its low prevalence there are limited data on therapeutics, particularly in refractory cases. Here, we discuss a case successfully managed with intravenous immunoglobulin and review the supporting literature.
\end{abstract}

(c) 2014 S. Karger AG, Basel

\section{Case Report}

A 36-year-old woman presented with a $12 \times 6 \mathrm{~cm}$ ulcer on her right leg (fig. 1). She stated that it had been present for 8 years and denied any history of malignancy, ulcerative colitis or rheumatic disease. Biopsy was diagnostic of pyoderma gangrenosum (PG) and prednisone $40 \mathrm{mg}$ daily was initiated. Due to incomplete response, she required several steroid-sparing agents, including dapsone, methotrexate, cyclosporine, mycophenolate, infliximab, adalimumab and azathioprine. Despite this, the ulcers persisted and the prednisone could not be tapered below $20 \mathrm{mg}$ daily. Intravenous immunoglobulin (IVIG) at $2 \mathrm{~g} / \mathrm{kg}$ monthly was initiated with nearly complete improvement (fig. 2). The prednisone was tapered to $5 \mathrm{mg}$ daily and IVIG was tapered to $2 \mathrm{~g} / \mathrm{kg}$ every 12 weeks. She has since done well on azathioprine and low-dose prednisone and has had no recurrent lesions. 


\begin{tabular}{l|l}
\hline DOI: $10.1159 / 000368824$ & $\begin{array}{l}\text { C 2014 S. Karger AG, Basel } \\
\text { www.karger.com/cde }\end{array}$ \\
\hline
\end{tabular}

Cafardi and Sami: Intravenous Immunoglobulin as Salvage Therapy in Refractory Pyoderma Gangrenosum: Report of a Case and Review of the Literature

\section{Discussion}

PG has an annual incidence of 3-10 per million persons, most commonly presents between 20 and 50 years of age and is more common in women. It is characterized by a neutrophilic infiltrate that is believed to be due to dysregulation of neutrophil function and altered innate immune responses. It frequently presents as painful ulcers that often follow surgery or trauma and is commonly misdiagnosed as infectious or ischemic lesions [1].

Initial therapy consists of corticosteroids, although steroid-sparing agents have been used, including dapsone, minocycline, methotrexate, cyclosporine, mycophenolate and TNF $\alpha$ inhibitors. IVIG is typically used after other treatments have failed. Careful wound care and avoidance of debridement and surgery is key [1].

We reviewed 20 cases of IVIG therapy in PG that are described in table 1 and table 2. Table 1 describes 13 cases that were treated with IVIG $2 \mathrm{~g} / \mathrm{kg}$ (except for two in whom the dose was 1.5 and $2.5 \mathrm{~g} / \mathrm{kg}$, respectively). 12 of these achieved complete or nearly complete remission; 2 had disease recurrence that required repeated courses of IVIG. Both instances of recurrent disease responded to repeat treatment with IVIG. Table 2 describes 7 patients treated with lower-dose IVIG $(0.5 \mathrm{~g} / \mathrm{kg})$. All had major or complete response, though all required continued corticosteroids, with 4 requiring additional immunosuppressive therapy.

Based on this case and our review of the literature, we believe that IVIG is a useful therapeutic option in refractory PG and can be considered in cases of resistance to or intolerance of standard immunomodulatory therapy.

This work was conducted at the University of Alabama, Birmingham, USA.

\section{Disclosure Statement}

The authors report no conflict on interest. There was no funding source.

\section{References}

1 Ruocco E, Sangiuliano S, Gravina AG, Miranda A, Nicoletti G: Pyoderma gangrenosum: an updated review. J Eur Acad Dermatol Venereol 2009;23:1008-1017.

-2 Gupta AK, Shear NH, Sauder DN: Efficacy of human intravenous immune globulin in pyoderma gangrenosum. J Am Acad Dermatol 1995;32:140-142.

-3 Cummins DL, Anhalt GJ, Monahan T, Meyerle JH: Treatment of pyoderma gangrenosum with intravenous immunoglobulin. Br J Dermatol 2007;157:1235-1239.

-4 Dirschka T, Kastner U, Behrens S, Altmeyer P: Successful treatment of pyoderma gangrenosum with intravenous human immunoglobulin. J Am Acad Dermatol 1998;39:789-790.

5 de Zwaan SE, Iland HJ, Damian DL: Treatment of refractory pyoderma gangrenosum with intravenous immunoglobulin. Australas J Dermatol 2009;50:56-59.

6 Hagman JH, Carrozzo AM, Campione E, Romanelli P, Chimenti S: The use of high-dose immunoglobulin in the treatment of pyoderma gangrenosum. J Dermatolog Treat 2001;12:19-22.

7 Meyer N, Ferraro V, Mignard MH, Adamski H, Chevrant-Breton J: Pyoderma gangrenosum treated with high-dose intravenous immunoglobulins: two cases and review of the literature. Clin Drug Investig 2006;26:541-546.

8 Suchak R, Macedo C, Glover M, Lawlor F: Intravenous immunoglobulin is effective as a sole immunomodulatory agent in pyoderma gangrenosum unresponsive to systemic corticosteroids. Clin Exp Dermatol 2007;32:205-207.

-9 Sinnya S, Hamza S: Pyoderma gangrenosum of the breast treated with intravenous immunoglobulin. J Dermatol Case Rep 2013;7:64-68.

10 Zhang XB, He YQ, Zhou H, Luo Q, Li CX: A case of pyoderma gangrenosum responding to high-dose intravenous immunoglobulin therapy. Chin Med J (Engl) 2006;119:1230-1232. 
Cafardi and Sami: Intravenous Immunoglobulin as Salvage Therapy in Refractory Pyoderma Gangrenosum: Report of a Case and Review of the Literature

11 Tamaki K, Nakazawa T, Mamehara A, Tsuji G, Saigo K, Kawano S, Morinobu A, Kumagai S: Successful treatment of pyoderma gangrenosum associated with myelodysplastic syndrome using high-dose intravenous immunoglobulin. Intern Med 2008;47:2077-2081.

12 Erfurt-Berge C, Herbst C, Schuler G, Bauerschmitz J: Successful treatment of pyoderma gangrenosum with intravenous immunoglobulins during pregnancy. J Cutan Med Surg 2012;16:205-207.

13 Kreuter A, Reich-Schupke S, Stucker M, Altmeyer P, Gambichler T: Intravenous immunoglobulin for pyoderma gangrenosum. Br J Dermatol 2008;158:856-857. 
Cafardi and Sami: Intravenous Immunoglobulin as Salvage Therapy in Refractory

Pyoderma Gangrenosum: Report of a Case and Review of the Literature

Table 1. Thirteen cases of IVIG therapy in PG (all IVIG courses were $2 \mathrm{~g} / \mathrm{kg}$ unless noted otherwise)

\begin{tabular}{|c|c|c|c|c|c|c|c|c|c|}
\hline $\begin{array}{l}\text { Age, } \\
\text { gender, } \\
\text { ref. }\end{array}$ & $\begin{array}{l}\text { Notable } \\
\text { past medical } \\
\text { history }\end{array}$ & Initial therapy & $\begin{array}{l}\text { Initial IVIG } \\
\text { courses }\end{array}$ & $\begin{array}{l}\text { Initial } \\
\text { response }\end{array}$ & $\begin{array}{l}\text { Re- } \\
\text { lapse }\end{array}$ & $\begin{array}{l}\text { Therapy } \\
\text { during IVIG } \\
\text { treatment }\end{array}$ & $\begin{array}{l}\text { Number of } \\
\text { further IVIG } \\
\text { courses }\end{array}$ & $\begin{array}{l}\text { Subsequen } \\
\text { response }\end{array}$ & Follow-up \\
\hline $\begin{array}{l}35, \mathrm{~F} \\
{[2]}\end{array}$ & insect bite & $\begin{array}{l}\text { PRD, DAP, CBT, } \\
\text { TAC, CSA, MPD }\end{array}$ & 2 (2 weeks apart) & $\begin{array}{l}\text { nearly } \\
\text { complete }\end{array}$ & no & PRD, CSA & none & $\mathrm{N} / \mathrm{A}$ & $\begin{array}{l}\text { clear at } \\
8 \text { months }\end{array}$ \\
\hline $\begin{array}{l}48, \mathrm{M} \\
{[3]}\end{array}$ & $\begin{array}{l}\text { adrenal } \\
\text { carcinoma, } \\
\text { pemphigus } \\
\text { vulgaris }\end{array}$ & $\begin{array}{l}\text { PRD, MMF, CSA, } \\
\text { DEX, THL }\end{array}$ & 1 & complete & yes & PRD, MMF & $\begin{array}{l}\text { yes, } \\
1 \text { course }\end{array}$ & complete & $\begin{array}{l}\text { clear at } \\
5 \text { months }\end{array}$ \\
\hline $\begin{array}{l}17, \mathrm{~F} \\
{[3]}\end{array}$ & none noted & $\begin{array}{l}\text { PRD, MPD, } \\
\text { MMF, CSA }\end{array}$ & $\begin{array}{l}\text { multiple (number } \\
\text { and interval not } \\
\text { given) }\end{array}$ & $\begin{array}{l}\text { nearly } \\
\text { complete }\end{array}$ & yes & CSA, MMF & yes & complete & $\begin{array}{l}\text { clear at } \\
15 \text { months }\end{array}$ \\
\hline $\begin{array}{l}37, \mathrm{~F} \\
{[4]}\end{array}$ & none noted & PRD, CSA & 4 (monthly) & complete & no & PRD & none & $\mathrm{N} / \mathrm{A}$ & $\begin{array}{l}\text { clear at } \\
4 \text { months }\end{array}$ \\
\hline $\begin{array}{l}55, \mathrm{M} \\
{[5]}\end{array}$ & $\mathrm{Ph}^{+} \mathrm{CML}$ & $\begin{array}{l}\text { PRD, MCN, DAP, } \\
\text { AZA, MMF, SSKI, } \\
\text { CSA }\end{array}$ & 3 (monthly) & complete & no & PRD & $\begin{array}{l}\text { yes, } \\
\text { indefinite }\end{array}$ & complete & $\begin{array}{l}\text { clear at } \\
8 \text { months }\end{array}$ \\
\hline $\begin{array}{l}81, \mathrm{M} \\
{[6]}\end{array}$ & trauma & CSA & 6 (monthly) & significant & no & MPD & none & $\mathrm{N} / \mathrm{A}$ & $\begin{array}{l}\text { clear at } \\
7 \text { months, then } \\
\text { died of cardiac } \\
\text { arrest }\end{array}$ \\
\hline $\begin{array}{l}58, \mathrm{M} \\
{[7]}\end{array}$ & $\begin{array}{l}\text { prostate } \\
\text { carcinoma }\end{array}$ & PRD, MCN & 6 (monthly) & significant & no & PRD & none & $\mathrm{N} / \mathrm{A}$ & $\begin{array}{l}\text { stable at } \\
12 \text { months }\end{array}$ \\
\hline $\begin{array}{l}66, M \\
{[7]}\end{array}$ & CMML & BTM, TAC, PRD & 6 (monthly) & significant & no & PRD & none & none & $\begin{array}{l}\text { stable at } \\
6 \text { months }\end{array}$ \\
\hline $\begin{array}{l}83, \mathrm{M} \\
{[8]}\end{array}$ & none noted & CBT, MCN, PRL & 5 (monthly) & significant & no & none & $\begin{array}{l}\text { yes, } 5 \text { courses } \\
\text { (bi-monthly) }\end{array}$ & complete & $\begin{array}{l}\text { stable at } \\
15 \text { months }\end{array}$ \\
\hline $\begin{array}{l}61, \mathrm{~F} \\
{[9]}\end{array}$ & $\begin{array}{l}\text { RA, insect } \\
\text { bite }\end{array}$ & PRD & 3 (monthly) & marked & no & PRD & no & $\mathrm{N} / \mathrm{A}$ & $\begin{array}{l}\text { stable } \\
\text { (unknown) }\end{array}$ \\
\hline $\begin{array}{l}62, \mathrm{~F} \\
{[10]}\end{array}$ & none noted & $\mathrm{CSA}, \mathrm{GC}$ & 4 (monthly)a & complete & no & PRD & no & $\mathrm{N} / \mathrm{A}$ & $\begin{array}{l}\text { stable at } \\
12 \text { months }\end{array}$ \\
\hline $\begin{array}{l}61, \mathrm{M} \\
{[11]}\end{array}$ & MDS & PRL, MPD & 2 (monthly) & marked & no & PRL, PRD & no & $\mathrm{N} / \mathrm{A}$ & $\begin{array}{l}\text { stable at } \\
3 \text { months }\end{array}$ \\
\hline $\begin{array}{l}26, \mathrm{~F} \\
{[12]}\end{array}$ & $\begin{array}{l}\text { twin } \\
\text { pregnancy }\end{array}$ & DAP, MPD & 4 (monthly) $^{\mathrm{b}}$ & significant & no & MPD & no & $\mathrm{N} / \mathrm{A}$ & $\begin{array}{l}\text { stable } \\
\text { (unknown) }\end{array}$ \\
\hline
\end{tabular}

The authors defined all clinical responses - there is no uniform standard for clinical response in PG.

$\mathrm{AZA}=$ Azathioprine; $\mathrm{BTM}=$ betamethasone (topical); $\mathrm{CBT}=$ clobetasol (topical); $\mathrm{CMML}=$ chronic myelomonocytic leukemia; $\mathrm{CSA}=$ cyclosporine A; DAP = dapsone; DEX = dexamethasone; GC = glucocorticoid (drug/route not specified); $\mathrm{MCN}$ = minocycline; MDS = myelodysplastic syndrome; $\mathrm{MMF}=$ mycophenolate mofetil; $\mathrm{MPD}=$ methylprednisolone; $\mathrm{Ph}{ }^{+} \mathrm{CML}=$ Philadelphia chromosome-positive chronic myeloid leukemia; $\mathrm{PRD}=$ prednisone; $\mathrm{PRL}$ = prednisolone; $\mathrm{RA}=$ rheumatoid arthritis; $\mathrm{SSKI}=$ potassium iodide; $\mathrm{TAC}=$ tacrolimus (topical); THL = thalidomide.

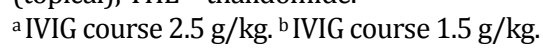


Cafardi and Sami: Intravenous Immunoglobulin as Salvage Therapy in Refractory Pyoderma Gangrenosum: Report of a Case and Review of the Literature

Table 2. Seven cases of IVIG therapy $(0.5 \mathrm{~g} / \mathrm{kg})$ in PG (all data are from Kreuter et al. [13])

\begin{tabular}{llll}
\hline $\begin{array}{l}\text { Age, } \\
\text { gender }\end{array}$ & Associated disease & $\begin{array}{l}\text { Concurrent } \\
\text { immunosuppression }\end{array}$ & $\begin{array}{l}\text { Treatment } \\
\text { response }\end{array}$ \\
\hline $47, \mathrm{~F}$ & ankylosing spondylitis & SGC & complete \\
$63, \mathrm{~F}$ & monoclonal gammopathy (IgA) & SGC, MMF, CSA & complete \\
$31, \mathrm{M}$ & ankylosing spondylitis & SGC, AZA, IFX & complete \\
$79, \mathrm{~F}$ & monoclonal gammopathy (IgA) & SGC & complete \\
$40, \mathrm{~F}$ & ulcerative colitis & SGC & major \\
$49, \mathrm{~F}$ & ulcerative colitis & SGC, AZA, CSA & major \\
$81, \mathrm{~F}$ & ulcerative colitis & SGC, CSA & complete \\
\hline
\end{tabular}

The authors defined all clinical responses - there is no uniform standard for clinical response in PG.

$\mathrm{AZA}=$ Azathioprine; $\mathrm{CSA}=$ cyclosporine A; IFX = infliximab; MMF = mycophenolate mofetil; $\mathrm{SGC}=$ systemic glucocorticoids.

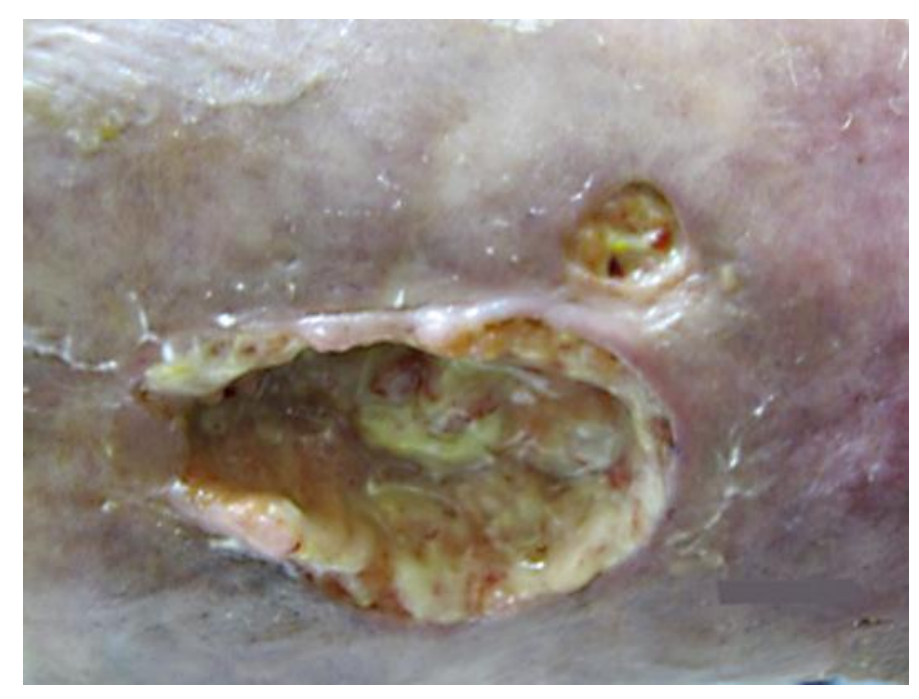

Fig. 1. PG lesion prior to IVIG therapy. 


\section{Case Reports in Dermatology}

Cafardi and Sami: Intravenous Immunoglobulin as Salvage Therapy in Refractory Pyoderma Gangrenosum: Report of a Case and Review of the Literature

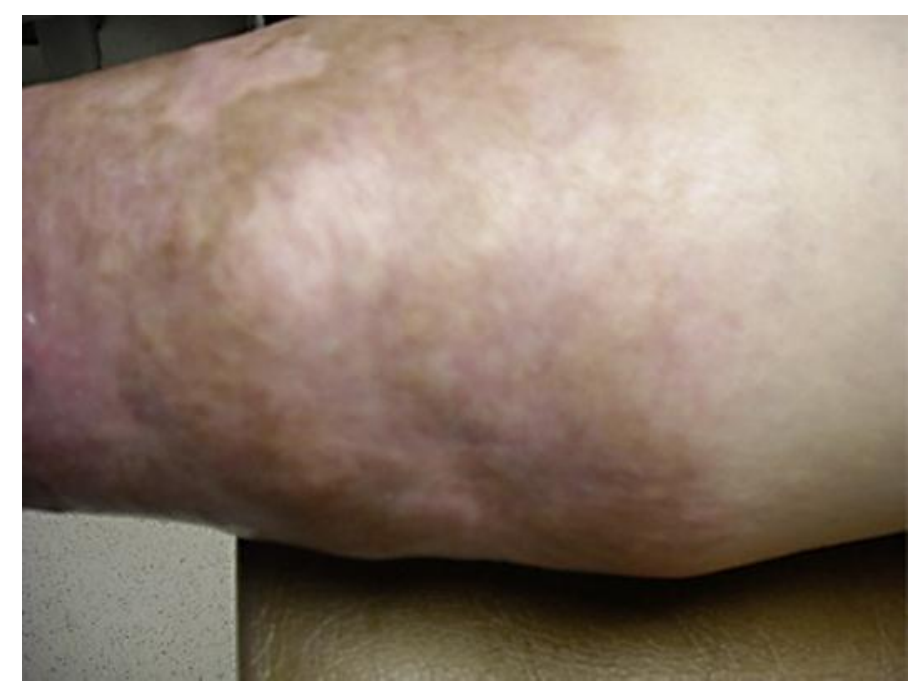

Fig. 2. PG lesion post IVIG therapy. 